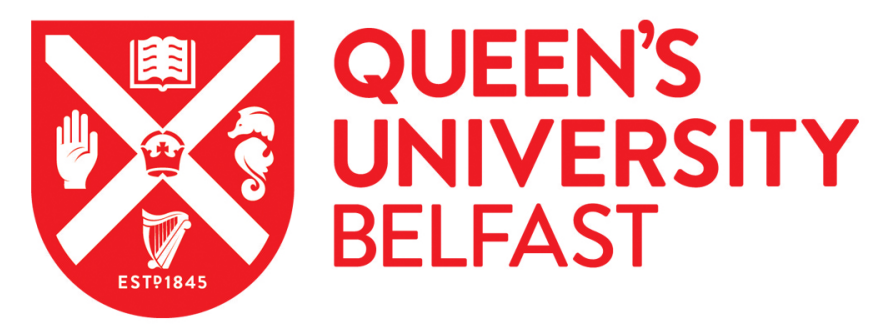

\title{
Wave-structure interaction of wave energy converters: A sensitivity analysis
}

Windt, C., Davidson, J., Schmitt, P., \& Ringwood, J. (2020). Wave-structure interaction of wave energy converters: A sensitivity analysis. ICE Proceedings - Engineering and Computational Mechanics.

https://doi.org/10.1680/jencm.19.00033

Published in:
ICE Proceedings - Engineering and Computational Mechanics

Document Version:

Peer reviewed version

Queen's University Belfast - Research Portal:

Link to publication record in Queen's University Belfast Research Portal

Publisher rights

Copyright 2020 ICE Publishing. This work is made available online in accordance with the publisher's policies. Please refer to any applicable terms of use of the publisher.

\section{General rights}

Copyright for the publications made accessible via the Queen's University Belfast Research Portal is retained by the author(s) and / or other copyright owners and it is a condition of accessing these publications that users recognise and abide by the legal requirements associated with these rights.

Take down policy

The Research Portal is Queen's institutional repository that provides access to Queen's research output. Every effort has been made to ensure that content in the Research Portal does not infringe any person's rights, or applicable UK laws. If you discover content in the Research Portal that you believe breaches copyright or violates any law, please contact openaccess@qub.ac.uk. 


\title{
Wave-structure interaction of wave energy converters: A sensitivity analysis
}

Christian Windt

Centre for Ocean Energy Research, Maynooth University, Maynooth, Co. Kildare, Ireland Josh Davidson

Department of Fluid Mechanics, Faculty of Mechanical Engineering, Budapest University of Technology and Economics, Hungary
Pál Schmitt

Marine Research Group, Queen's University Belfast, Northern Ireland John V. Ringwood

Centre for Ocean Energy Research, Maynooth University, Maynooth, Co. Kildare, Ireland

\begin{abstract}
Measurement uncertainties are inevitable during physical wave tank tests. Therefore, when validating a numerical wave tank against experiments, knowledge of the uncertainties in the physical experiments, and the sensitivity of the body dynamics to such uncertainties, is crucial. Specifically, the inertial properties, as well as the location of the centre of mass, which are challenging to measure accurately, can have a significant influence on the system dynamics. Based on the test cases of the CCP-WSI Blind Test Series 2, this paper presents a sensitivity analysis of the agreement between experimental and numerical data sets to the quality of the incident, focused wave, as well as the inertial properties and the location of the centre of mass. A significant influence on the modelled system dynamics can be observed when changing the system parameters, resulting in a variation of the observed error of up to 80 percentage points for pitch motion.
\end{abstract}

\section{Introduction}

Numerical wave tanks (NWTs) are virtual test-beds for the analysis of wave structure interaction (WSI). The continued increase in the availability of high-performance computing resources has enabled NWTs to become an essential part of offshore/coastal engineering and a valuable design tool for wave energy converters (WECs). NWTs allow the investigation of different designs and arbitrary tank layouts, with the ability to passively measure any variable in all locations throughout the tank. Generally, NWTs can achieve different levels of fidelity, at different levels of computational cost (Penalba et al., 2017). At the lower end of the fidelity spectrum, NWTs based on linear potential flow assume linear conditions (small wave amplitude and body motions) and are computationally efficient tools for early stage design or parametric studies. However, the linear assumptions are pushed beyond the limits of validity when large body motions or non-linear free surface deformations occur. In contrast, at the higher end of the fidelity spectrum, CFDbased NWTs inherently capture all relevant hydrodynamic nonlinearities and can produce high-fidelity, high resolution data sets, but require substantially more computation.

It is well-known that the application of CFD-based NWTs relies on both verification and validation to ensure the accuracy of the numerical results (Roache, 1998) and, thus, should be a part of every NWT experiment. Using well established CFD software (e.g. OpenFOAM, Star-CCM+, etc.), verification embraces the quantification of discretisation errors (spatially and temporally). Validation, in contrast, covers the comparison of the simulated results to reference data. Generally, three different validation strategies can be identified, where CFD results are compared to: (1) analytical results; (2) third-party numerical results; (3) experimental data.

Using experimental data allows for a direct comparison against measured physical reality and is the most prominent method used during WEC model validation (Windt et al., 2018). However, experimental reference data are typically only available from scale model testing in a physical wave tank. These physical wave tank tests may suffer from scaling effects, measurement uncertainty, and peculiarities of the test facility. Therefore, validating a CFD-based NWT model against experimental data risks the danger of drawing false conclusions when experimental inaccuracies and wave tank artefacts are not taken into account.

\subsection{Related studies}

Schmitt and Elsässer (2015a) provide quantitative comparisons between experimental and simulation results for an oscillating wave surge converter. The authors highlight the complexity and potential inaccuracies of the particular experimental setup, identifying perturbations in the exciting waves, due to reflections and other perturbations, as a significant contribution to the observed differences between the numerical and experimental data. 
Palm et al. (2016) identify the influence of physical model inaccuracies on CFD-based NWT model validation, for a novel coupled mooring analysis methodology. The inaccuracies embrace manufacturing tolerances of physical models or material properties. Prasad et al. (2017) details measurement uncertainties of $\pm 1 \%$ to $\pm 2 \%$, caused by the instrumentation.

Dai et al. (2019) validate a numerical model of a fixed oscillating water column, against physical wave tank tests, to analyse scale effects. Two different scales (with a scale ratio of 1:3) are considered in both, an experimental and numerical test frameworks. For the experimental measurement uncertainty, the authors follow the recommendations in (ITTC, 2008). Comparing the response amplitude operators (RAOs) and the captured power, between experimental and numerical results, discrepancies of up to $15 \%$ are observed when including measurement uncertainty.

$\mathrm{Xu}$ et al. (2019) perform experimental and numerical analysis of a two-body, floating point-absorber type WEC, in operational and survival conditions. For both conditions, independent experimental test campaigns, in different test facilities and at different scales, were performed. For the survivability test cases, discrepancies for the surge and pitch RAOs are attributed to a mismatch in the model geometries and the centre of mass (CoM). Quantification of the model geometrical discrepancies is, however, omitted.

Windt et al. (2020) validate a 1:5 scale NWT model of the Wavestar WEC, for power production assessment, finding discrepencies between phyical and numerical results for the surface elevation, body motion, pressure on the hull, and power take-off force, of the order of $\mathcal{O}(10 \%)$. It is highlighted that the validation of complex physical systems requires exact knowledge of all system characteristics for the formulation of assumptions in the numerical model. This detailed knowledge is difficult to acquire and inaccuracies may influence the achievable order of accuracy for validation studies.

\subsection{Objectives}

This paper presents a sensitivity analysis, for the simulated dynamics of two point-absorber type WECs, concerning the accuracy of the incident wave and crucial system parameters, such as: inertial properties and the location of the CoM. The case study, used for the sensitivity analysis, is based on the Blind Test Series 2 of the Collaborative Computational Project in Wave Structure Interaction (CCP-WSI) (Ransley et al., 2019, n.d.). The numerical results are compared to the recently disclosed experimental data, recorded for Blind Test Series 2.

The remainder of this paper is organised as follows. Section 2 provides details on the physical wave tank tests and presents the different inertial properties and the locations of the CoM, considered in the sensitivity analysis. Section 3 details the equivalent NWT setup, including a description of the numerical wave generation and absorption methods. The results for different test cases, considered in the sensitivity analysis, are then discussed in Section 4. Finally, conclusions are drawn in Section 5.

\section{Physical wave tank tests}

For the CCP-WSI Blind Test Series 2, physical wave tank tests were conducted in the ocean basin of the COAST laboratory at the University of Plymouth. The ocean basin is $32.1 \mathrm{~m}$ long, $15.65 \mathrm{~m}$ wide, $3 \mathrm{~m}$ deep, and is equipped with a flap-type wave maker and an absorbing beach. A schematic of the physical wave tank, including the locations of the wave probes, is depicted in Figure 1. The test campaign includes WSI, as well as wave-only experiments.
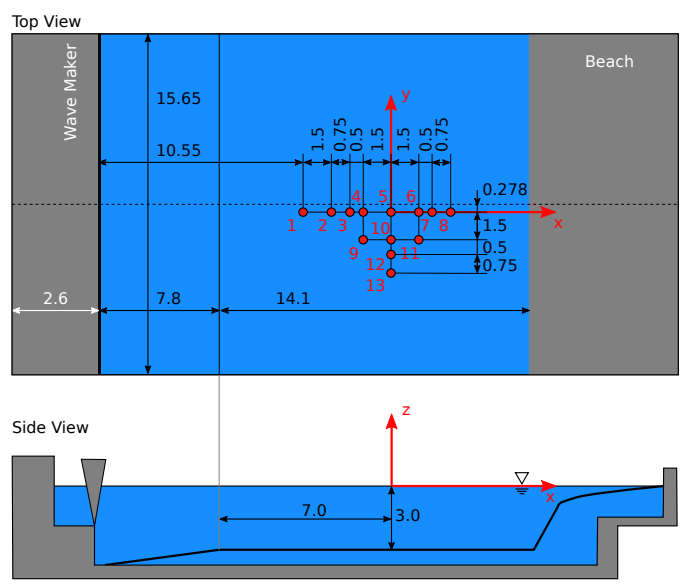

Figure 1. Schematic (not to scale) of the physical wave tank (dimensions in [m]). The red circles indicate the wave probe locations. For the WSI experiments, wave probe 5 is replaced by the WEC structure.

\subsection{Input waves}

The test series considers three focused waves of varying steepness, 1BT2 - 3BT2, whose characteristics are listed in Table 1. The experimentally measured surface elevation, for each wave at the focus location, is plotted in Fig. 2.

Table 1. Characteristics of the three considered focused waves

\begin{tabular}{ccccccc}
\hline Wave & $A n[\mathrm{~m}]$ & $f p[\mathrm{~Hz}]$ & $d[\mathrm{~m}]$ & $H s[\mathrm{~m}]$ & $\lambda[\mathrm{m}]$ & $k A[-]$ \\
\hline \hline 1BT2 & 0.25 & 0.3578 & 3.0 & 0.274 & 11.35 & 0.129 \\
2BT2 & 0.25 & 0.4 & 3.0 & 0.274 & 9.41 & 0.161 \\
3BT2 & 0.25 & 0.4382 & 3.0 & 0.274 & 7.99 & 0.193
\end{tabular}

$A n$ : Peak wave amplitude; $f p$ : Peak wave frequency; $d$ : water depth; $H s$ : significant wave height; $\lambda$ : wave length; $k$ : wave number

\subsection{WEC structures}

Two different WEC structures are considered, W1 and W2, resembling moored point absorber type devices. Both structures have axisymmetric, cylindrical, geometries. All relevant physical properties are shown in Figure 3. The mooring of the structures 


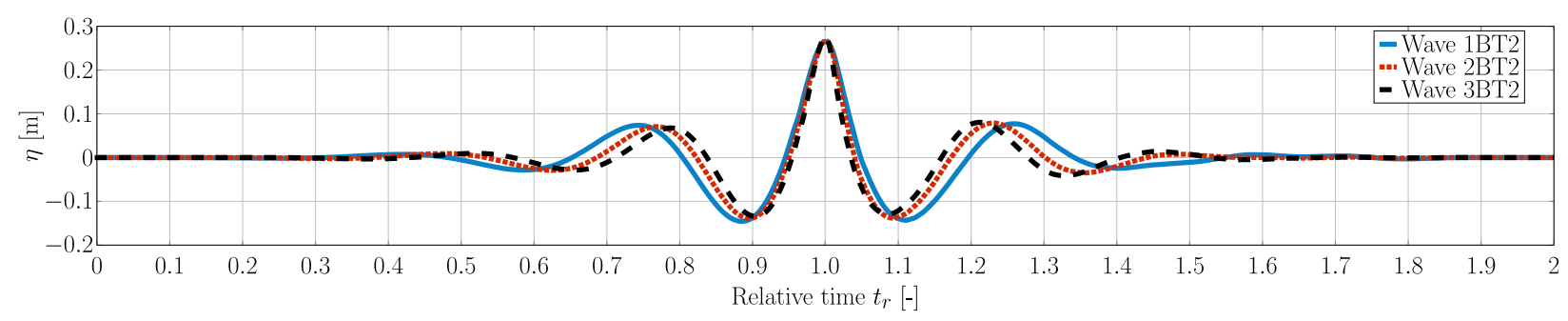

Figure 2. Experimentally measured surface elevation, $\eta$, of waves $1 \mathrm{BT} 2-3 \mathrm{BT} 2$, at the focal location (wave probe 5).

is implemented with a linear spring, with a stiffness of $67 \mathrm{~N} \mathrm{~m}^{-1}$, connecting the device with the tank floor.

As stated in Ransley et al. (n.d.), measurement uncertainties can not be ruled out, specifically for the inertial properties, as well as the exact location of the CoM. To analyse the sensitivity of the body dynamics and, thereby, the agreement between the numerical and experimental data set, to the uncertainty in the inertia and the location of the CoM, simulations are performed in which an uncertainty of $\pm 10 \%$ is added to Ixx, Iyy, and Izz, and the vertical location of the CoM. The considered values are listed in Table 2. (a) W1

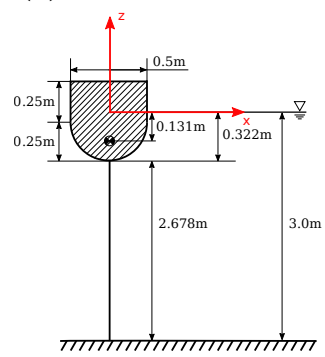

(b) W2

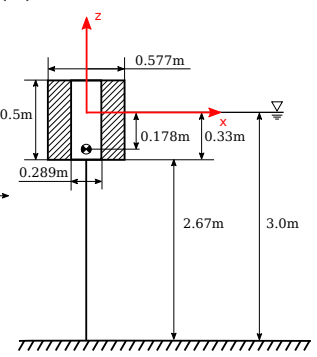

Figure 3. Schematic of the considered WEC structures.

Table 2. Inertial properties and the vertical location of the CoM for the sensitivity analysis

\begin{tabular}{lcccc} 
& \multicolumn{3}{c}{ Inertia [kg m$\left.{ }^{2}\right]$} & CoM [m] \\
& Ixx & Iyy & Izz & \\
\hline \hline W1 & & & & \\
+10\% Inertia & 1.782 & 1.782 & 1.257 & -0.131 \\
-10\% Inertia & 1.458 & 1.458 & 1.029 & -0.131 \\
+10\% CoM & 1.620 & 1.620 & 1.143 & -0.118 \\
-10\% CoM & 1.620 & 1.620 & 1.143 & -0.144 \\
\hline \hline W2 & & & & \\
+10\% Inertia & 3.916 & 3.916 & 3.628 & -0.178 \\
-10\% Inertia & 3.204 & 3.204 & 2.968 & -0.178 \\
+10\% CoM & 3.560 & 3.560 & 3.298 & -0.160 \\
-10\% CoM & 3.560 & 3.560 & 3.298 & -0.196 \\
\hline
\end{tabular}

\section{NWT Setup}

The CFD-based NWT in this study is based on the open-source CFD software, OpenFOAM 4.1. The hydrodynamics in the CFDbased NWT are captured by solving the incompressible RANS equations, describing the conservation of mass and momentum:

(1) $\quad \nabla \cdot \mathbf{U}=0$

(2) $\frac{\partial \rho \mathbf{U}}{\partial t}+\nabla \cdot \rho \mathbf{U U}=-\nabla p+\nabla \cdot(\mu \nabla \mathbf{U})+\rho \mathbf{f}_{b}$,

respectively. Here, $t$ denotes time, $\mathbf{U}$ is the fluid velocity, $p$ the fluid pressure, $\rho$ the fluid density, $\mathbf{f}_{b}$ the external forces such as gravity, and $\mu$ the dynamic viscosity (Ferziger et al., 2002). Note that, based on the findings in Windt et al. (2019b), laminar flow conditions are assumed throughout this study. The water wave advection is captured via the volume of fluid method, proposed by Hirt and Nichols (1981), following

$$
\frac{\partial \alpha}{\partial t}+\nabla \cdot(\mathbf{U} \alpha)+\nabla \cdot\left[\mathbf{U}_{\mathbf{r}} \alpha(1-\alpha)\right]=0,
$$

and

(4) $\Phi=\alpha \Phi_{\text {water }}+(1-\alpha) \Phi_{\text {air }}$,

where $\alpha$ denotes the volume fraction of water, $\mathbf{U}_{\mathbf{r}}$ is the compression velocity (Berberović et al., 2009), and $\Phi$ is a specific fluid quantity. To measure the surface elevation, the iso-surface of the volume fraction $\alpha=0.5$ is recorded.

The body motion is solved via Newton's 2nd law of motion, within the sixDoFRigidBodyMotionSolver in the OpenFOAM framework. The motion solver provides a set of motion restraints, allowing the implementation of a linear spring to account for the mooring of the device. The resulting body motion is accommodated in the numerical domain through mesh morphing, delivering computationally efficient and accurate results (Windt et al., 2019a). Note that the symmetry of the problem is exploited and only half of the physical wave tank is modelled numerically. A symmetry boundary condition is employed in the x,z-plane, where $\mathrm{x}$ points in the wave propagation direction, and $-\mathrm{z}$ towards the tank floor. This symmetry condition introduces constraints on the body motion, only allowing motion in three degrees of freedom (DoFs), i.e. heave, surge, and pitch. Additional information on the NWT settings are listed in Table 3. 
Table 3. Additional information on the NWT settings

\begin{tabular}{ll}
\hline Pressure-velocity coupling & PIMPLE (2 corrector loops) \\
Observed maximum Courant number & 0.47 \\
Dynamic mesh motion method & Spherical linear interpolation \\
& (SLERP) algorithm ${ }^{1}$ \\
accelerationRelaxation & 0.7 \\
\hline${ }^{1}$ For more information, the interested reader is referred to \\
(Windt et al., 2019a)
\end{tabular}

\subsection{Problem discretisation}

Convergence studies on the spatial and temporal problem discretisation have been performed, using three different discretisation levels. Table 4 shows the results for the convergence studies, based on the WSI simulations for wave $1 \mathrm{BT} 2$, including the convergence type and the discretisation uncertainty, $\bar{U}$ (Eça and Hoekstra, 2014). Based on the results a fixed time step size of $0.002 \mathrm{~s}$ is used. The minimum cell size in the interface region and around the WEC structure is 10 cells per significant wave height of wave 1BT2. The maximum aspect ratio in the interface region is 2 . Three refinement levels are used to reach the cell size in the interface region. The total cell count for the NWT is approx. one million cells. A screen shot of the mesh layout is shown in Figure 4.

Table 4. Results of the spatial and temporal convergence study based on the WSI simulations for wave 1BT2

\begin{tabular}{cccc}
\hline & Max. heave & Max. surge & Max. pitch \\
\hline \hline$\Delta z=5 \mathrm{CPHs}$ & $0.226 \mathrm{~m}$ & $0.466 \mathrm{~m}$ & 0.293 \\
$\Delta z=10 \mathrm{CPHs}$ & $0.236 \mathrm{~m}$ & $0.353 \mathrm{~m}$ & 0.333 \\
$\Delta z=20 \mathrm{CPHs}$ & $0.238 \mathrm{~m}$ & $0.341 \mathrm{~m}$ & 0.332 \\
Conv. Type & Monotone & Monotone & Oscillatory \\
$\bar{U}$ & $0.6 \%$ & $0.6 \%$ & $0.03 \%$ \\
\hline \hline$\Delta t=0.004 \mathrm{~s}$ & $0.239 \mathrm{~m}$ & $0.338 \mathrm{~m}$ & 0.338 \\
$\Delta t=0.002 \mathrm{~s}$ & $0.238 \mathrm{~m}$ & $0.341 \mathrm{~m}$ & 0.332 \\
$\Delta t=0.001 \mathrm{~s}$ & $0.238 \mathrm{~m}$ & $0.342 \mathrm{~m}$ & 0.329 \\
Conv. Type & Monotone & Monotone & Monotone \\
$\bar{U}$ & $0.8 \%$ & $0.6 \%$ & $1.9 \%$
\end{tabular}

\subsection{Numerical wave generation and absorption}

Different numerical wave makers are available to generate and absorb waves in a CFD-based NWT (Miquel et al., 2018). Herein, the impulse source method, proposed by Schmitt et al. (2019), is employed, where a source term, $\mathbf{r} \rho \mathbf{a}_{w m}$, is added to the RANS momentum equation (2), yielding:

$$
\frac{\partial \rho \mathbf{U}}{\partial t}+\nabla \cdot \rho \mathbf{U U}=-\nabla p+\nabla \cdot(\mu \nabla \mathbf{U})+\rho \mathbf{f}_{b}+\mathbf{r} \rho \mathbf{a}_{w m}
$$

The location of the wave maker zone is defined by $\mathbf{r}=1$, with $\mathbf{r}=0$ elsewhere in the domain (see Figure 5 (a)). $\mathbf{a}_{w m}$ is the field variable acting as an acceleration input to the wave maker, determined herein via an iterative calibration method (see Section 3.2.1). The location of the impulse source has been chosen based on

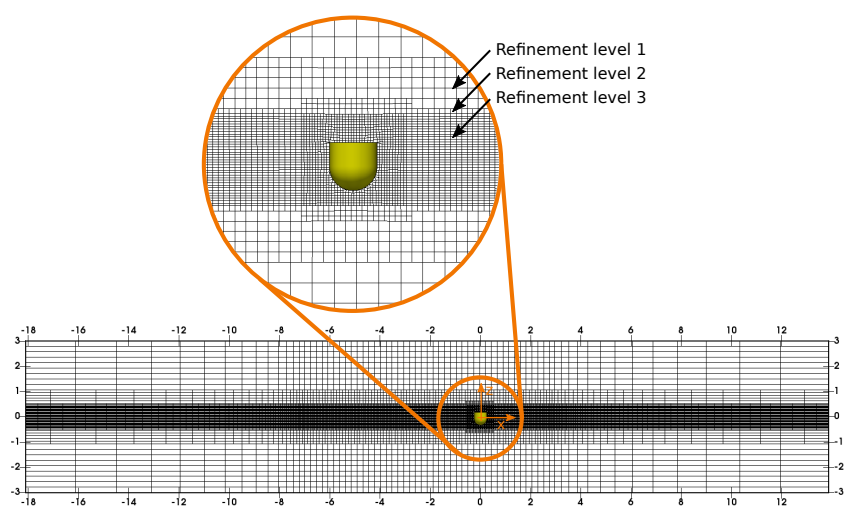

Figure 4. Mesh layout in the numerical domain. Cell stretching is applied towards the far field boundaries of the domain. To achieve the desired cell size in the interface region, three refinement levels are implemented. The overall domain dimension are $31.95 \times 6 \times 7.8 \mathrm{~m}(\mathrm{LxH} \times \mathrm{W})$.

the distance between the end of the slope towards the paddle type wave maker and the WEC structure, i.e. $7 \mathrm{~m}$ (see Figure 1).

For wave absorption, a numerical beach, proposed by Schmitt and Elsässer (2015b), is implemented. Introducing the dissipation term, $\mathbf{S} \rho \mathbf{U}$, to the RANS momentum equation (2), yields:

$$
\frac{\partial \rho \mathbf{U}}{\partial t}+\nabla \cdot \rho \mathbf{U U}=-\nabla p+\nabla \cdot(\mu \nabla \mathbf{U})+\rho \mathbf{f}_{b}+\mathbf{S} \rho \mathbf{U}
$$

The variable field $\mathbf{S}$ controls the dissipation strength, with a value of zero in the simulation zone, which then gradually increases, following an analytical expression, over a defined beach length, to a maximum damping factor, $S_{\max }$. Based on the findings in Windt et al. (2019c), the beach length is set to $\lambda_{1 \mathrm{BT} 2}$, i.e. the longest wave length. Different $S_{\max }$ values are evaluated, by comparing the reflection coefficient, $R$, calculated using the three point method (Mansard and Funke, 1980). Sufficient wave absorption $(R \leq$ $3.5 \%$ ) is achieved with $S_{\max }=3 \mathrm{~s}^{-1}$ for all tested waves. A screen shot of the NWT, showing the field variable $\mathbf{S}$, is depicted in Figure 5 (b).

\subsubsection{Wave maker calibration}

To generate the desired target wave at a specific location within the NWT, a calibration procedure is used to determine the required impulse source input $\mathbf{a}_{w m}$. For the initial results, submitted to Blind Test Series 2 (Windt et al., 2019b), the calibration procedure is adapted from a standard spectral analysis method based on (Masterton and Swan, 2008). The calibration method comprises the following steps (for a graphical representation, see Figure 6):

1. Define a target wave time series, $\eta_{t}(t)$ 
(a)

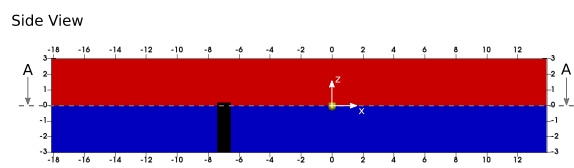

(b)

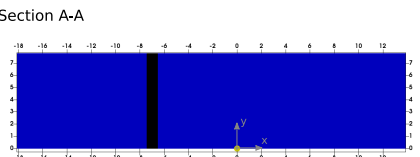

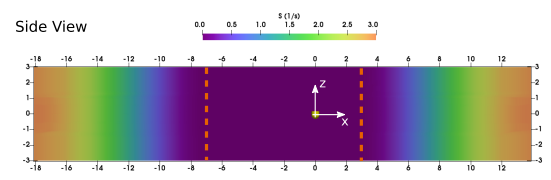

Figure 5. 2D screen shots of the CFD-based NWT showing (a) the water and air phase (blue and red colour code, respectively), and the impulse source (black colour code). The WEC structure (yellow colour code) is located at $(x, y, z)=(0,0,0)$; (b) the gradually increasing damping factor $\mathbf{S}$ of the numerical beach. The simulation zone is situated between the dashed orange lines, where $\mathbf{S}=0$.

2. Fast Fourier Transform (FFT) $\eta_{t}(t)$, to obtain the amplitudes and phases for each frequency component

3. Define an initial time series for $\mathbf{a}_{w m, 1}(t)$, serving as input to the OpenFOAM simulation

4. FFT $\mathbf{a}_{w m, 1}(t)$, to obtain the amplitudes and phases for each frequency component of $\mathbf{a}_{w m, 1}$

5. Run the OpenFOAM simulation for calibration iteration $i$, using $\mathbf{a}_{w m, i}(t)$ and extraction of the resulting surface elevation, $\eta_{r, i}(t)$, at the specific location.

6. FFT $\eta_{r, i}(t)$, to obtain the amplitudes and phases for each frequency component of $\eta_{r, i}(t)$

7. Correct the amplitude components of $\mathbf{a}_{w m, i}(t)$, by scaling with the ratio of the $\eta_{t}(t)$ and $\eta_{r, i}(t)$ amplitude components

8. Correct the phase components of $\mathbf{a}_{w m, i}(t)$, by summing the phase with the difference between the $\eta_{t}(t)$ and $\eta_{r, i}(t)$ phase components

9. Construct $\mathbf{a}_{w m, i+1}(t)$, using the Inverse Fourier Transform of the corrected amplitude and phase components

10. Repeat Steps 5 - 9, either for a maximum number of iterations, or until a threshold for the error metric, between the $\eta_{t}(t)$ and $\eta_{r, i}(t)$, is reached.

In the present paper, the initial calibration method has been updated, to achieve a better agreement between the target and the resulting wave. The improved agreement between the target and resulting wave is presented and discussed in Section 4.1. The calibration method now comprises the following steps (for a graphical representation, see Figure 7):

1. Define of a target wave time series, $\eta_{t}(t)$

2. Extend $\eta_{t}(t)$ using zero padding for increased frequency resolution

3. Compute the frequency domain equivalent of $\eta_{t}(t)$

4. Define an initial time series for $\mathbf{a}_{w m, 1}(t)$

5. Extend $\mathbf{a}_{w m, 1}(t)$ using zero padding for increased frequency resolution

6. Compute the frequency domain equivalent of $\mathbf{a}_{w m, 1}(t)$

7. Run the OpenFOAM simulation for calibration iteration $i$, using $\mathbf{a}_{w m, i}(t)$ and extraction of the resulting surface elevation, $\eta_{r, i}(t)$, at the specific location.

8. Compute the frequency domain equivalent of $\eta_{r, i}(t)$
9. Compute the transfer function $H_{i}(j \omega)$ from $\mathbf{a}_{w m, i}(j \omega)$ to $\eta_{r, i}(j \omega)$

10. Compute the frequency domain equivalent of the new source input $\mathbf{a}_{w m, i+i}(j \omega)$ with $H_{i}^{-1}(j \omega)$

11. Construct $\mathbf{a}_{w m, i+1}(t)$, using the Inverse Fourier Transform on $\mathbf{a}_{w m, i+i}(j \omega)$

12. Filter $\mathbf{a}_{w m, i+1}(t)$, using a cut-off frequency of $1 \mathrm{~Hz}$

13. Repeat Steps 7 - 13, either for a maximum number of iterations, or until a threshold for the error metric between the $\eta_{t}(t)$ and $\eta_{r, i}(t)$ is reached.

\section{Results \& Discussion}

In the following, the numerical results are presented and compared to the experimental data from CCP-WSI Blind Test Series 2. Quantification of the agreement between the data sets is evaluated via the normalised root mean square error (nRMSE), following:

(7) $\mathrm{nRMSE}=\sqrt{\frac{\sum_{i=1}^{n}\left[y_{\exp }(i)-y_{\mathrm{num}}(i)\right]^{2}}{n}} \frac{100 \%}{\sigma_{\exp }}$,

where $y_{\exp }$ denotes the experimental quantity, $y_{\text {num }}$ is the corresponding numerical quantity, and $n$ defines the signal length via the number of samples. For consistency with Ransley et al. (n.d.), the RMSE is normalised by the standard deviation of the particular experimental data set, $\sigma_{\exp }$.

\subsection{Waves-only}

Before considering WSI simulations, wave-only tests were simulated. Figure 8 shows the time traces of the target waves, together with the numerical results, stemming from the initial and the updated calibration method ${ }^{*}$.

Qualitatively, significant improvement in the numerical results can be observed in Figure 8, for the peak-succeeding troughs and the tail of the focused wave, induced by the updated calibration method.

\footnotetext{
* Note that this paper only includes surface elevation time traces for the wavemaker inputs used in the WSI simulations. For more information on the improvement of the agreement between the target and the resulting wave within the calibration procedure, the interested reader is referred to (Windt et al., 2019b).
} 


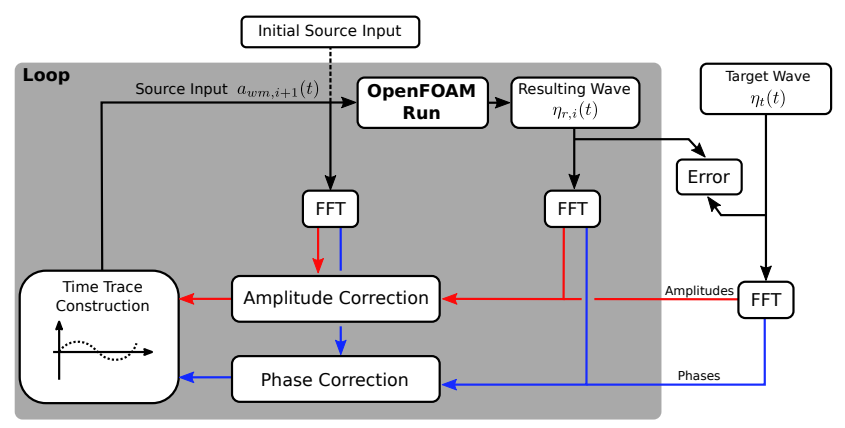

Figure 6. Initial calibration scheme for the impulse source input based on (Schmitt et al., 2019)

For a quantitative comparison, Table 5 lists the nRMSE values, for waves 1BT2-3BT2, achieved using the initial and the updated calibration method. The largest drop (14 percentage points) in the nRMSE can be identified for wave 3BT2. It is worth noting that the quality of the resulting wave (with the updated calibration method), quantified by the nRMSE, does not show a dependency on the wave steepness.

Table 5. nRMSE for the waves-only tests

\begin{tabular}{ccc} 
Wave & Initial calibration [\%] & Updated calibration [\%] \\
\hline \hline 1BT2 & 22.44 & 10.89 \\
2BT2 & 23.29 & 16.78 \\
3BT2 & 26.22 & 12.66 \\
\hline
\end{tabular}

\subsubsection{Wave propagation}

Any calibration procedure, whether initial or updated, runs the risk of producing a well-captured wave at the specific location considered in the calibration, without properly capturing the wave propagation. To that end, Figure 9 shows the time traces of the surface elevation measured at wave probes $1,3,5$, and 8 (see Figure 1), for waves 1BT2-3BT2, generated with the initial and the updated calibration methods.

The agreement between the experimental and numerical surface elevation follows the trend identified from Figure 8. The peakpreceding part of the time trace is well captured for the waves at all wave probes, using either the initial or the updated calibration method. More significant differences between the initial and the updated calibration method can be observed towards the end of the time traces. Especially at wave probe 1, high frequency components are induced by the initial calibration method, while a closer match between the updated calibration method and the experimental data is achieved. Overall, wave propagation in the physical wave tank is consistently well captured in the NWT.

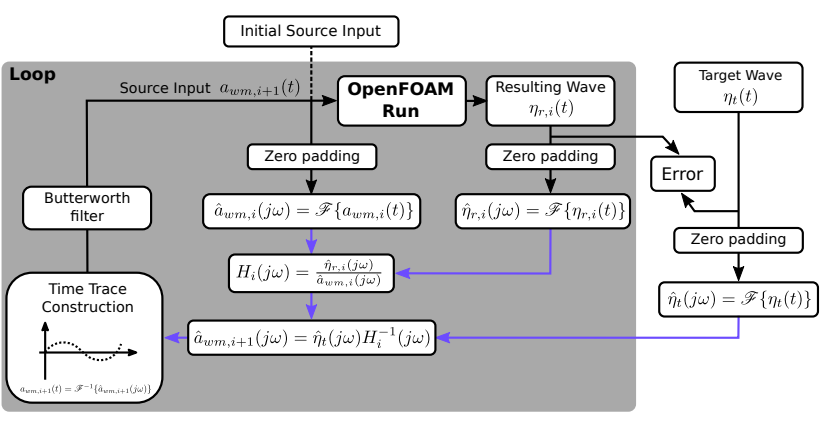

Figure 7. Updated calibration scheme for the impulse source input

\subsection{WSI}

The sensitivity of the body dynamics to inaccuracies in the surface elevation, inertial properties, and the vertical CoM location is analysed in this section, by means of a comparison between experimental reference data and the numerical results.

\subsubsection{Structure $\mathrm{W} 1$}

For WEC structure W1, the time traces of the heave, surge, and pitch motion, as well as the mooring force and surface elevation for waves 1BT2-3BT2, are shown in Figure 10. Specifically, two cases are shown: results with the waves generated using the initial and the updated calibration method ${ }^{\dagger}$. A qualitative comparison between the different time traces reveals an overall good agreement between the experimental data set and both numerical data sets. The largest deviations can be observed towards the end of the signals, after the main crest and, specifically, for the surge and pitch DoFs. No significant qualitative difference in agreement can be observed between the three different waves.

Comparing the two numerical data sets from the initial submission and the updated calibration method, it can be observed that the improved agreement between the experimental and numerical surface elevation, after updating the calibration method, manifests itself by an improved agreement of the body dynamics. Specifically for heave motion and mooring force, better agreement is achieved towards the end of the signal, thereby following the trend of the surface elevation, which, in fact, is consistent with the findings in (Ransley et al., n.d.). For completeness, Figure 11 shows the spectral density function for heave, surge, and pitch motion, as well as mooring force and surface elevation for waves 1BT23BT2. The plots for the heave motion, mooring force, and surface elevation most clearly indicate the improvement in the agreement between the experimental and numerical data after updating the calibration method. Considering the time traces, the overall largest deviations are observed for the surge and pitch motion. The spectral

${ }^{\dagger}$ Note, in the following, the results with the wave generated from the initial calibration method represent the results submitted to the Blind Test Series 2 (Windt et al., 2019b) 


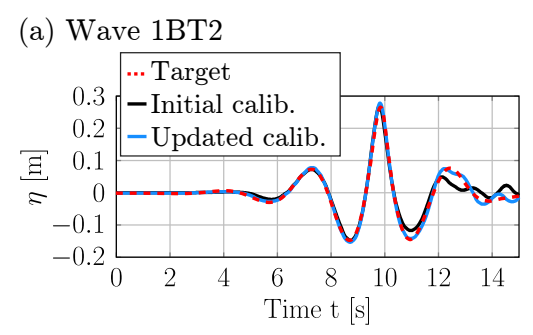

(b) Wave 2BT2

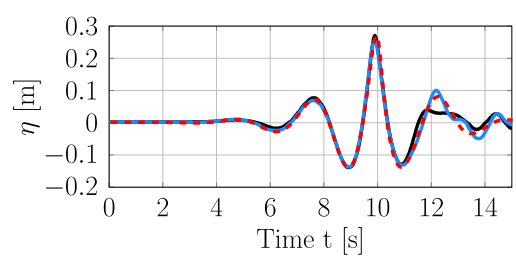

(c) Wave 3BT2

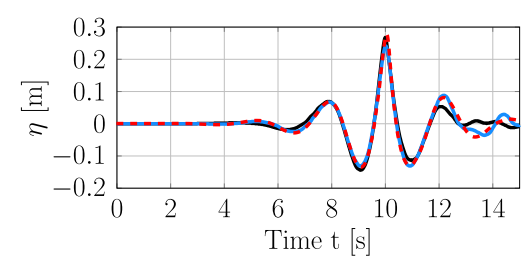

Figure 8. Target and resulting surface elevation for waves 1BT2 (a), 2BT2 (b), and 3BT2 (c).

(a) $1 \mathrm{BT} 2$

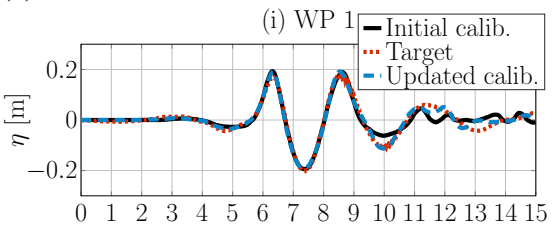

(ii) WP 3

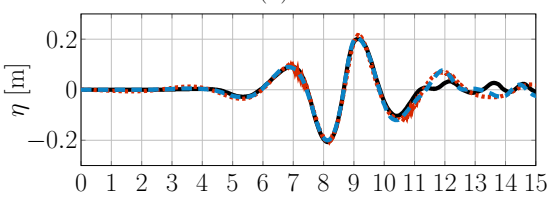

(iii) WP 5

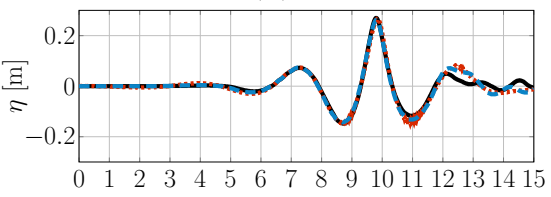

(iv) WP 8

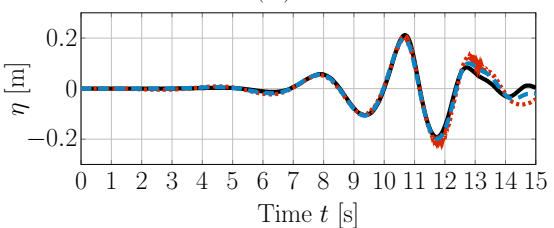

(b) $2 \mathrm{BT} 2$

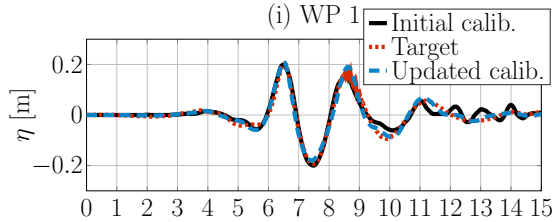

(ii) WP 3

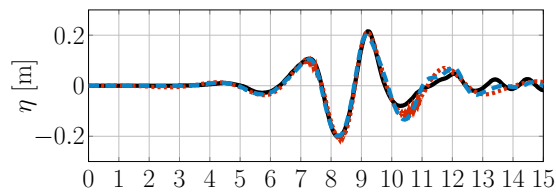

(iii) WP 5

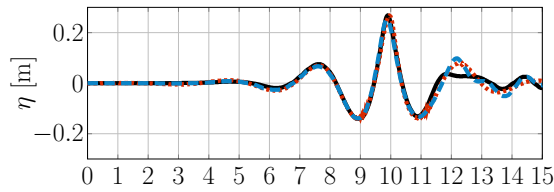

(iv) WP 8

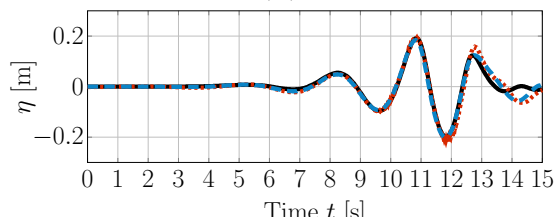

(c) $3 \mathrm{BT} 2$

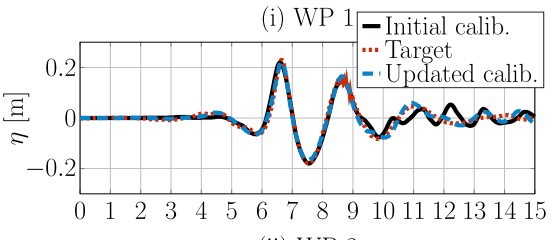

(ii) WP 3

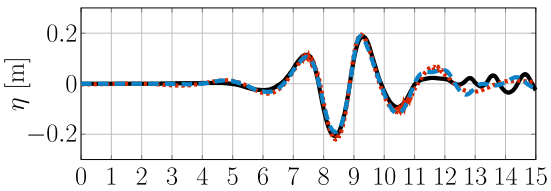

(iii) WP 5

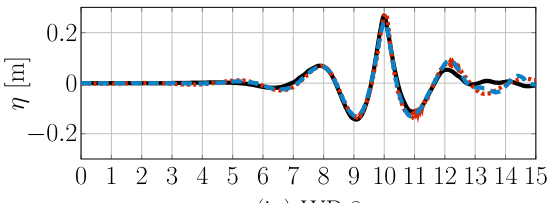

(iv) WP 8

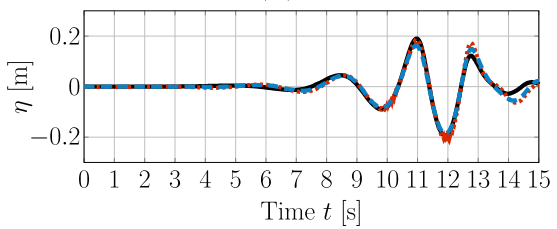

Figure 9. Experimental and numerical surface elevation time traces for waves 1BT2-3BT2, measured at wave probes (WPs) 1, 3, 5, and 8 (see Figure 1)

density function reveals a mismatch, in the peak period of the pitch motion, between the numerical and experimental data, specifically for waves 2BT2 and 3BT2.

For a quantitative comparison, Figure 14 (a), (c), and (e) show the nRMSE for the surface elevation, heave, surge, and pitch motion, as well as the mooring force, for waves 1BT2-3BT2, respectively. The bar graphs show that the improved agreement between the experimental and numerical results, for the surface elevation, induced by updated calibration method, has a positive influence on the heave motion (maximum drop of the nRMSE from $27 \%$ to $15 \%$ for wave $3 \mathrm{BT} 2$ ) and the mooring force (maximum drop from $26 \%$ to $14 \%$ for wave $3 \mathrm{BT} 2$ ). Regarding the sensitivity to changed inertial properties or CoM location, the heave motion and mooring force show negligible dependency on the changed properties. At this point, it should be noted that the sensitivity analysis to the physical WEC properties is carried out considering the updated calibration method.

Compared to the heave motion and the mooring force, the surge motion generally shows larger errors of the order of $30-40 \%$. Again, this trend is consistent with the findings in (Ransley et al., n.d.). The time traces in Figure 10 indicate that the increased error mainly stems from the deviation at the tail of the signal. Interestingly, the surge motion also shows a stronger dependency on the agreement with the experimental results on the change of 
the physical properties. Together with the strong dependency of the nRMSE values on the physical properties for the pitch motion, a coupling between these two DoFs is indicated. Furthermore, it is striking that, for waves $1 \mathrm{BT} 2$ and $2 \mathrm{BT} 2$, the error between the experimental and numerical results with the improved incident wave increases, compared to the initial submission. Only for wave $3 \mathrm{BT} 2$ the error in the surge motion decreases when improving the agreement of the surface elevation, thereby following the trend of the heave motion and mooring force.

As expected, the largest sensitivity to variations in different physical properties can be observed for pitch motion $(20 \% \leq \mathrm{nRMSE} \leq$ $138 \%$ ). For waves 1BT2 and 3BT2, similar trends can be observed. The smallest errors are achieved either with a decreased inertia (30\% for $1 \mathrm{BT} 2$ and $22 \%$ for 3BT2) or by lowering the CoM $(36 \%$ for $1 \mathrm{BT} 2$ and $20 \%$ for $3 \mathrm{BT} 2$ ). Using the same inertial properties and location of the CoM, as provided by Blind Test Series 2 organisers, and only improving the fidelity of the surface elevation leads to an increase in the error for the pitch motion $(+24 \%$ for wave $1 \mathrm{BT} 2 ;+3 \%$ for wave $3 \mathrm{BT} 2$ ). The time traces in Figure 10 (a) indicated that the increased error for wave 1BT2 mainly stems from a divergence in the phase after approx. 12s. Interestingly, between $12 \mathrm{~s} \leq t \leq 15 \mathrm{~s}$, significantly better agreement between the experimental and numerical surface elevation can be observed for the surface elevation from the updated calibration method.

For wave 2BT2, the pitch motion does not follow the previously observed trend, showing the smallest error of the different test cases for the initial inertial properties, as well as location of the CoM, and the improved surface elevation (28\%). The time trace in Figure 10 (b) indicates better agreement in phase between the experimental data and the numerical data, with improved surface elevation, compared to the initial submission, while a very similar agreement in amplitude between the two data sets can be observed for the three different waves. This highlights the sensitivity of the RMSE to phase shifts and, further, the importance of investigating both time traces and nRMSE to get a complete view of the agreement between experimental and numerical data. Nonetheless, no explanation was found for the cause of the observed phase shift for the pitch motion.

\subsubsection{Structure $\mathrm{W} 2$}

For WEC structure W1, Figure 12 shows the time traces of the heave, surge, and pitch motion, as well as the mooring force and surface elevation for waves 1BT2-3BT2, for structure W2 and the cases of the initial calibration and the improved surface elevation. The corresponding spectral density functions are shown in Figure 13. Generally, similar trends as for W1 can be observed. Notably, relatively large differences between the experimental and both numerical data sets can be observed for the pitch motion. While, as for all motion data and the mooring force, the main peak and the preceding trough is well captured, significant differences in the phase and amplitude become visible towards the end of the pitch motion time traces. This is highlighted in the plots of the SDF, as well as the values of the nRMSE.

Comparing the nRMSE values from the initial calibration to the cases with the improved surface elevation and the initial inertial properties and location of the CoM, a consistent drop in the error can be observed for all waves (up to 13 percentage points for wave 1BT2). Compared to structure W1, the nRMSE values for the pitch motion are consistently higher for structure W2, for both the initial and updated surface elevation. However, by lowering the vertical position of the CoM, the error in the pitch motion can be significantly reduced (down to $13 \%$ ), thereby falling in the same range as for structure W1. Generally, an overall consistent trend can be observed for the pitch motion for all waves, whereby an increased inertia or a raised CoM significantly increases the observed nRMSE (up to $131 \%$ for wave 2BT2).

Regarding the nRMSE for the heave motion, as well as the mooring force, similar error values are achieved for structures W2 as for $\mathrm{W} 1$, following the same trend as indicated in Section 4.2.1. With an improved surface elevation, the error in the heave motion and mooring force is decreased and negligible scatter between the cases $\pm 10 \%$ inertia or $\pm 10 \%$ CoM can be observed, indicating an overall strong coupling between the surface elevation, heave motion and mooring force. For the surge motion, more significant scatter between the cases can be observed. Contrary to structure W1, significant drops in the error can be observed when lowering the vertical location of the CoM (minimum nRMSE $=10 \%$ for wave $2 \mathrm{BT} 2$ ).

Overall, the results for structure W1 indicate a more consistent sensitivity of the modelled body dynamics to the physical device properties, resulting in an overall best agreement (nRMSE $\leq 21 \%$ ) between the experimental and numerical results for the cases of a lower CoM.

\section{Conclusions}

This paper presents a sensitivity analysis of the agreement between the experimental and numerical body dynamics, by means of the nRMSE, on the quality of the incident wave, as well as the crucial system parameters, i.e. inertial properties and the location of the vertical $\mathrm{CoM}$. From the presented results, the following conclusions can be drawn:

The sensitivity of the nRMSE to the quality of the surface elevation can be observed to be significant for both WEC structures.

- A strong coupling between the surface elevation, heave motion, and mooring force can be observed. Furthermore, Coupling can also be observed between the surge and pitch motion.

- The heave motion and mooring force are generally unaffected by the changed system parameters. 
The pitch motion shows strong sensitivity to the quality of the surface elevation and, most significantly, to changes in system parameters.

- For W1, changing the inertia or the location of the CoM has comparable effects.

W2 shows larger sensitivity to the change in the location of the CoM, compared to changes in the inertia

- The study highlights the importance of accurate measurements of the physical system properties, including error margins.

- This study neglects the potential effects of drag on the mooring cable, which could add additional uncertainty to the model behaviour.

\section{Acknowledgements}

This paper is based upon work supported by Science Foundation Ireland under Grant No. 13/IA/1886. The research reported in this paper was furthermore supported by the Higher Education Excellence Program of the Ministry of Human Capacities in the frame of Water science \& Disaster Prevention research area of Budapest University of Technology and Economics (BME FIKPVÍZ).

\section{REFERENCES}

Berberović E, van Hinsberg NP, Jakirlić S, Roisman IV and Tropea C (2009) Drop impact onto a liquid layer of finite thickness: Dynamics of the cavity evolution. Physical Review E 79: 036306-1 - 036306-15.

Dai S, Day S, Yuan Z and Wang H (2019) Investigation on the hydrodynamic scaling effect of an owc type wave energy device using experiment and cfd simulation. Renewable Energy 142: 184-194.

Eça $L$ and Hoekstra M (2014) A procedure for the estimation of the numerical uncertainty of cfd calculations based on grid refinement studies. Journal of Computational Physics 262: 104-130.

Ferziger JH, Perić M and Street RL (2002) Computational methods for fluid dynamics, vol. 3. Springer.

Hirt CW and Nichols BD (1981) Volume of Fluid (VOF) Method for the Dynamics of Free Boundaries. Journal of Computational Physics 39: 201-225.

ITTC (2008) Recommended Procedures and Guidelines: Uncertainty Analyssis Instrument Calibration. Technical report, ITTC.

Mansard E and Funke E (1980) The measurement of incident and reflected spectra using a least squares method. In Proceedings of the International Conference on Coastal Engineering, Sydney, pp. 154-172.

Masterton S and Swan C (2008) On the accurate and efficient calibration of a 3D wave basin. Ocean Engineering 35: 763-773.

Miquel A, Kamath A, Alagan Chella M, Archetti R and Bihs H (2018) Analysis of different methods for wave generation and absorption in a CFD-based numerical wave tank. Journal of Marine Science and Engineering 6(2): 73.

Palm J, Eskilsson C, Paredes GM and Bergdahl L (2016) Coupled mooring analysis for floating wave energy converters using CFD: Formulation and validation. International Journal of Marine Energy 16: 83-99.

Penalba M, Giorgi G and Ringwood JV (2017) Mathematical modelling of wave energy converters: a review of nonlinear approaches. Renewable and Sustainable Energy Reviews 78: 1188-1207.

Prasad DD, Ahmed MR, Lee YH and Sharma RN (2017) Validation of a piston type wave-maker using numerical wave tank. Ocean Engineering 131: 57-67.

Ransley E, Yan S, Brown S, Hann M, Graham D, Windt C, Schmitt P, Davidson J, Ringwood J, Musiedlak PH, Wang J, Wang J, Ma Q, Xie Z, Zhang N, Zheng X, Giorgi G, Chen H, Lin Z, Qian L, Ma Z, Bai W, Chen Q, Zang J, Ding H, Cheng L, Zheng J, Gu H, Gong X, Liu Z, Zhuang Y, Wan D, Bingham $\mathrm{H}$ and Greaves D (n.d.) A blind comparative study of focused wave interactions with floating structures (CCP-WSI Blind Test Series 3). International Journal of Offshore and Polar Engineering in review.

Ransley E, Yan S, Brown S, Mai T, Graham D, Ma Q, Musiedlak PH, Engsig-Karup A, Eskilsson C, Li Q, Wang J, Xie Z, Sriram V, Stoesser T, Zhuang Y, Li Q, Wan D, Chen G, Chen H, Qian L, Ma Z, Mingham C, Causon D, Gatin I, Jasak H, Vukevi V, Downie S, Higuera P, Buldakov E, Stagonas D, Chen Q, Zang J and Greaves D (2019) A blind comparative study of focused wave interactions with a fixed FPSO-like structure (CCP-WSI Blind Test Series 1). International Journal of Offshore and Polar Engineering 29: 113-127.

Roache PJ (1998) Verification and validation in computational science and engineering, vol. 895. Hermosa Publisher Albuquerque, NM.

Schmitt P and Elsässer B (2015a) On the use of OpenFOAM to model oscillating wave surge converters. Ocean Engineering 108: 98 - 104.

Schmitt P and Elsässer B (2015b) A review of wave makers for 3D numerical simulations. In MARINE 2015 - Computational Methods in Marine Engineering VI, Rome, pp. 437-446.

Schmitt P, Windt C, Davidson J, Ringwood J and Whittaker T (2019) The efficient application of an impulse source wave maker to CFD simulations. Journal of Marine Science and Engineering 7(3).

Windt C, Davidson J, Chandar DD, Faedo N and Ringwood JV (2019a) Evaluation of the overset grid method for control studies of wave energy converters in openfoam numerical wave tanks. Journal of Ocean Engineering and Marine Energy : $1-16$.

Windt C, Davidson J, Ransley EJ, Greaves D, Jakobsen M, Kramer M and Ringwood JV (2020) Validation of a cfd-based numerical wave tank model for the power production assessment of the 
(a) Wave $1 \mathrm{BT}$
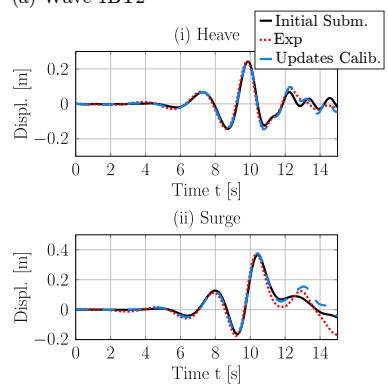

(iii) Pitch

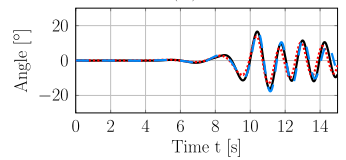

(iv) Mooring Force

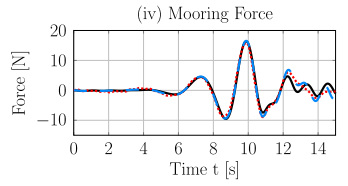

(v) Surface elevation

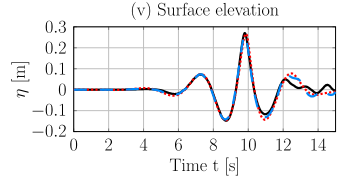

(a) Wave 2BT2
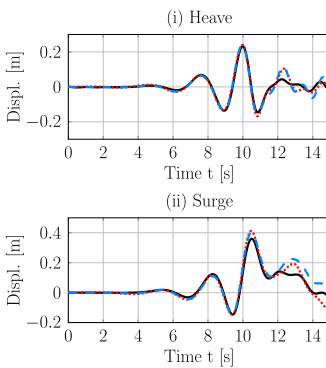

(iii) Pitch
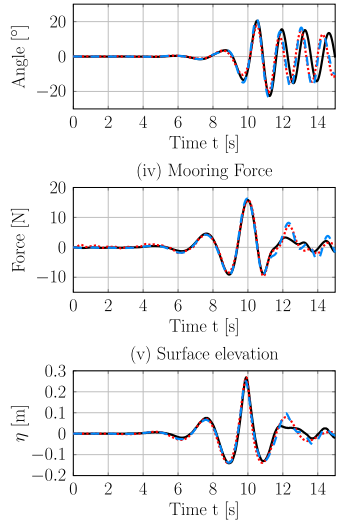

(a) Wave 3BT2
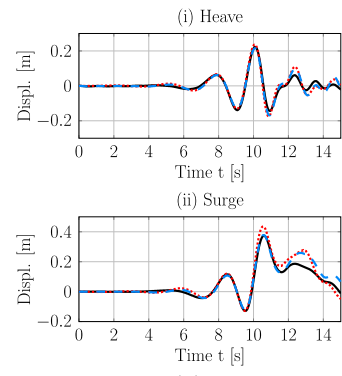

(iii) Pitch

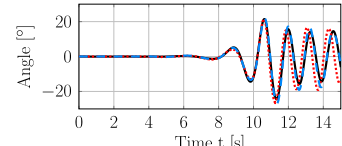

(iv) Mooring Force
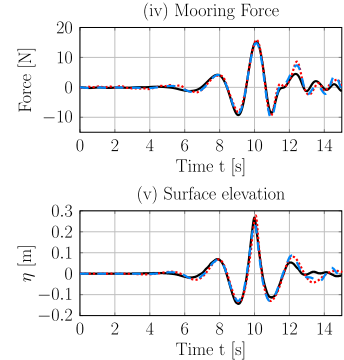

Figure 10. Time traces of the heave, surge, and pitch motion, as well as the mooring force and surface elevation for W1

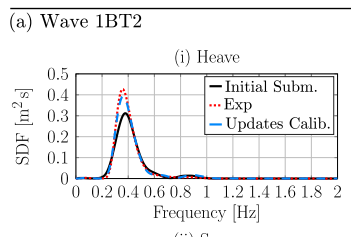

(ii) Surge
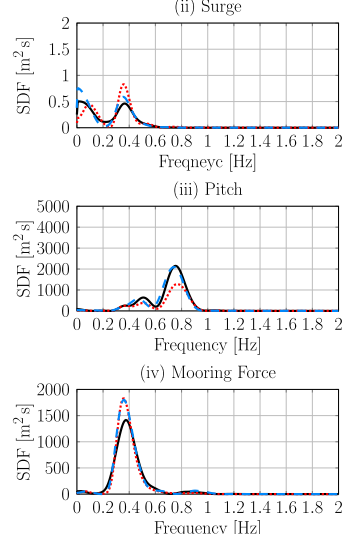

(v) Surface elevation

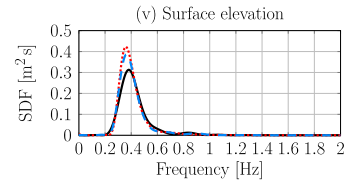

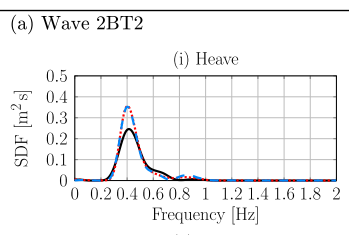
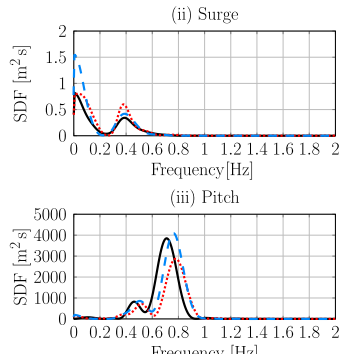

Frequency $[\mathrm{Hz}]$

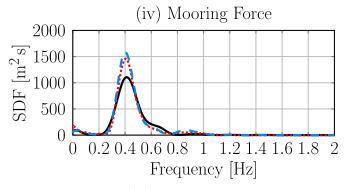

(v) Surface elevation

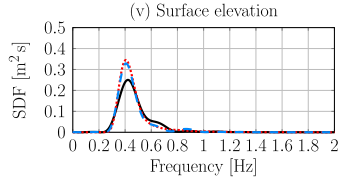

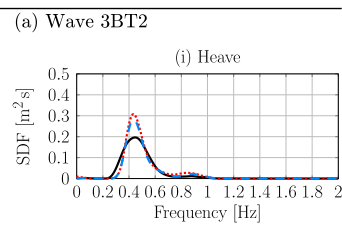

(ii) Surge

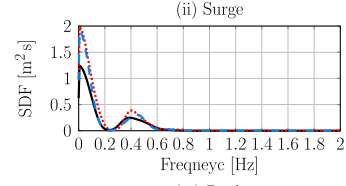

(iii) Pitch
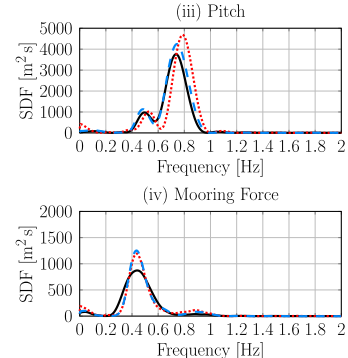

(v) Surface elevation

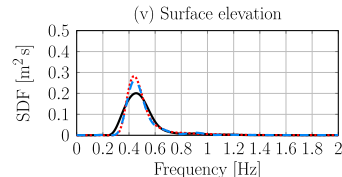

Figure 11. SDF of the heave, surge, and pitch motion, as well as the mooring force and surface elevation for W1 
(a) Wave $1 \mathrm{BT}$
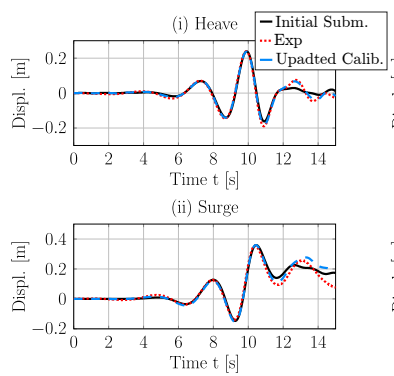

(iii) Pitch

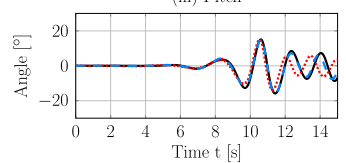

(iv) Mooring Force

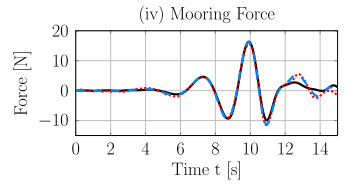

(v) Surface elevation

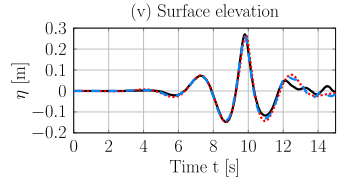

(a) Wave $2 \mathrm{BT} 2$
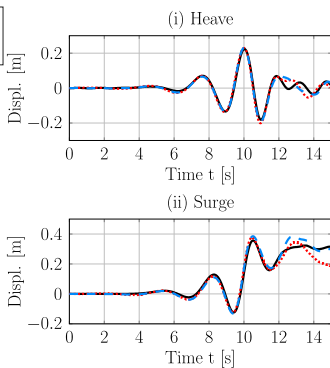

(iii) Pitch
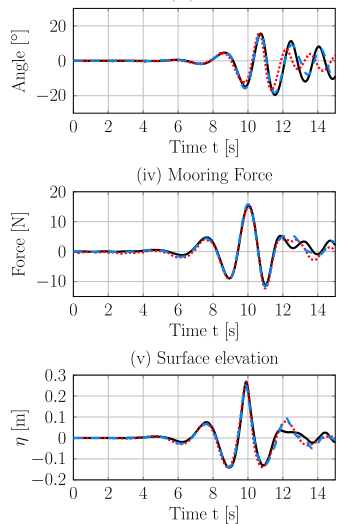

(a) Wave $3 \mathrm{BT} 2$
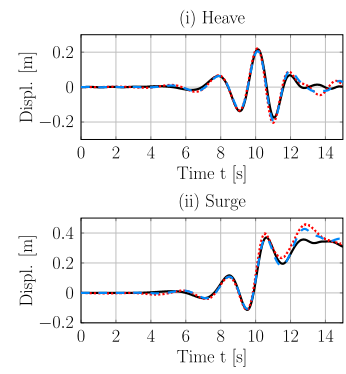

(iii) Pitch

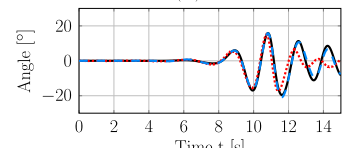

(iv) Mooring Force

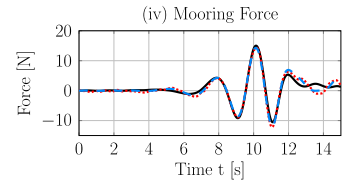

(v) Surface elevation

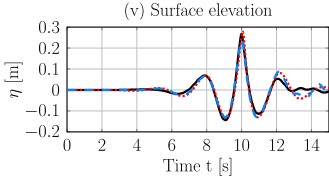

Figure 12. Time traces of the heave, surge, and pitch motion, as well as the mooring force and surface elevation for W2
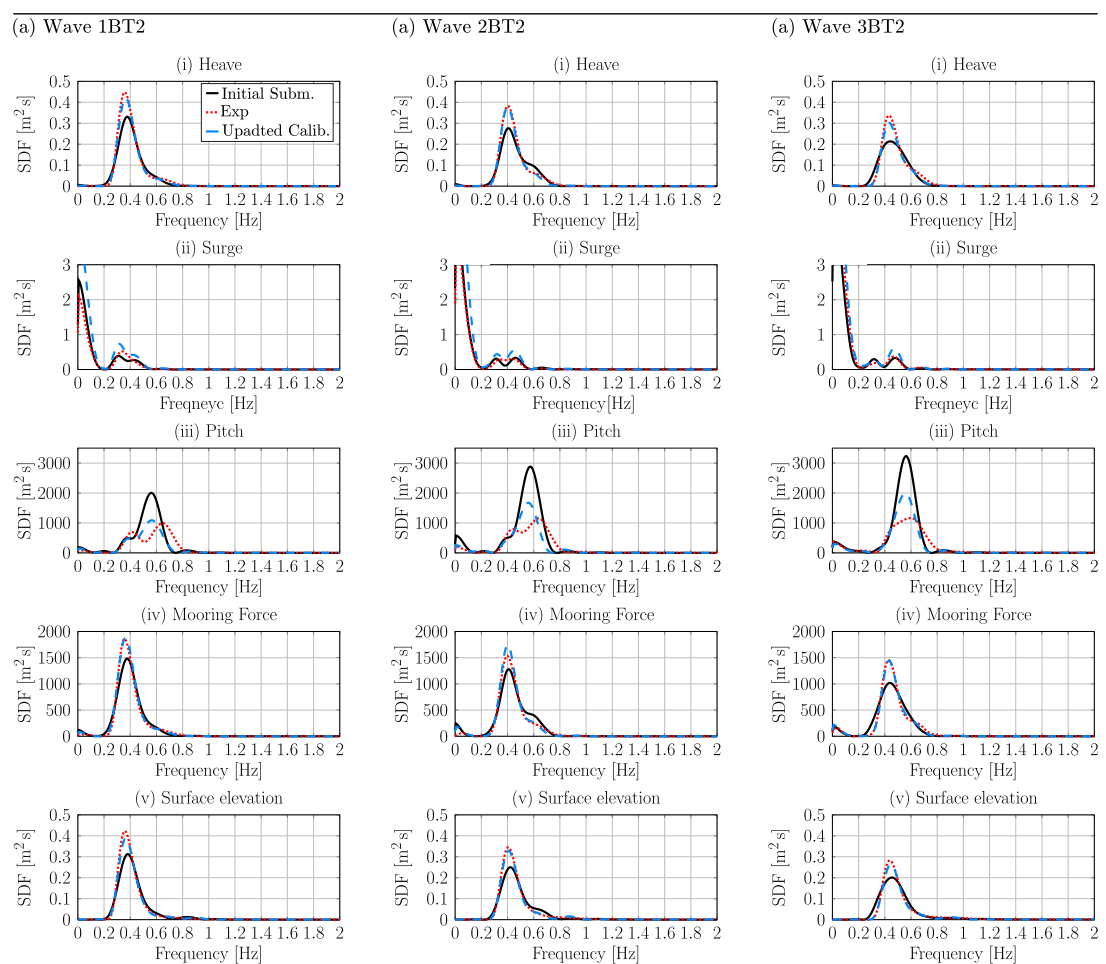

Figure 13. SDF of the heave, surge, and pitch motion, as well as the mooring force and surface elevation for W2 
(a) W1 1BT2
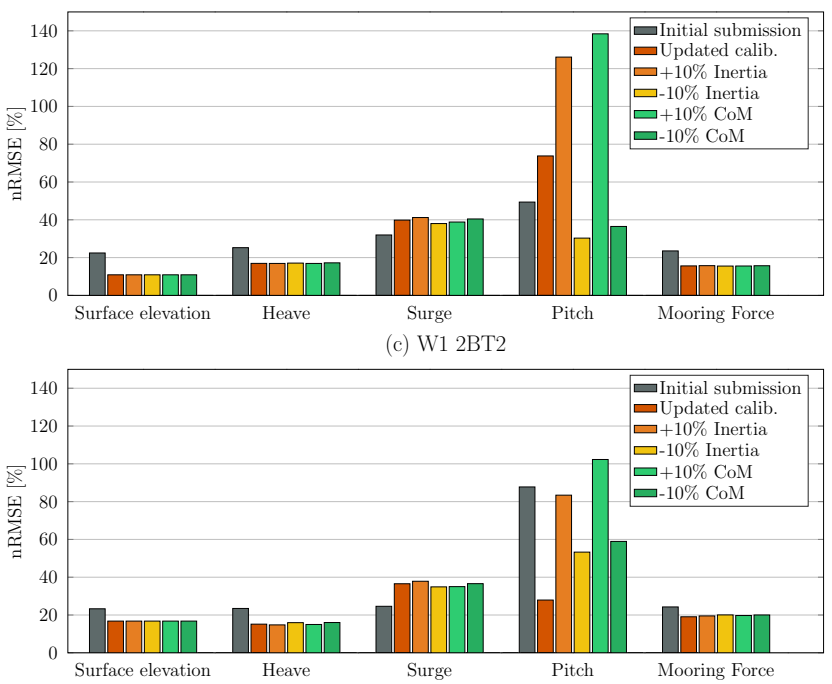

(e) W1 3BT2

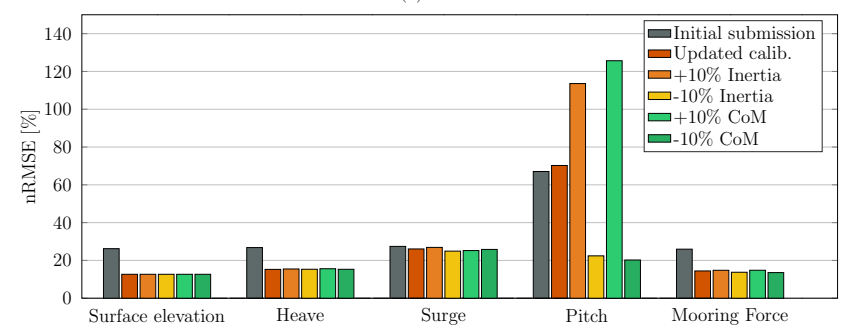

(b) W2 1BT2

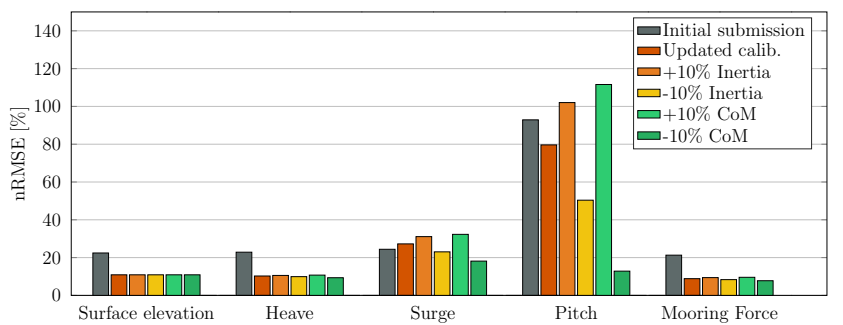

(d) W2 2BT2

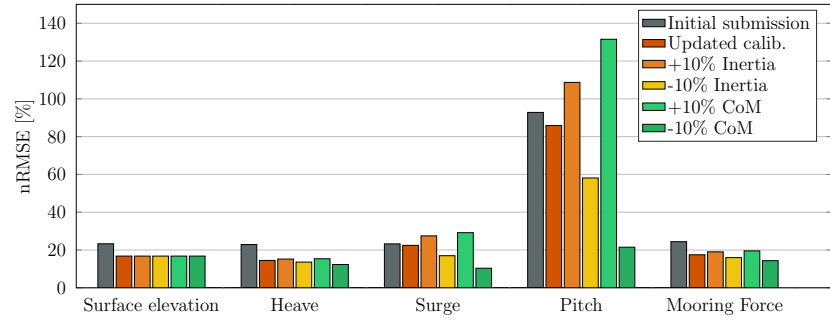

(f) W2 3BT2

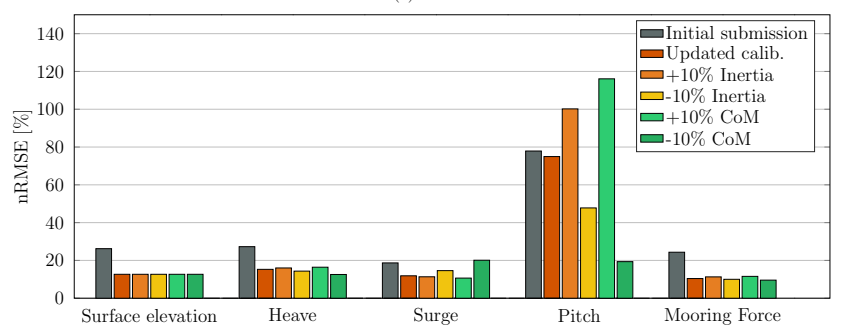

Figure 14. nRMSE between the experimental and numerical data for WEC structure W1 and W2 exposed to waves 1BT2-3BT2 for the different test cases considered in the sensitivity analysis.

wavestar ocean wave energy converter. Renewable Energy 146: 2499-2516.

Windt C, Davidson J, Schmitt P and Ringwood JV (2019b)

Contribution to the CCP-WSI Blind Test Series 2: CFD-based numerical wave tank experiments employing an impulse source wave maker. In Proceedings of the 13th European Wave and Tidal Energy Conference, Naples, pp. 1265-1 - 1265-10.

Windt C, Davidson J, Schmitt P and Ringwood JV (2019c) On the assessment of numerical wave makers for CFD simulations.
Journal of Marine Science and Engineering 7(2).

Windt C, Faedo N, Penalba M and Ringwood J (2018) Evaluation of energy maximising control systems for the wavestar wave energy converter. In Proceedings of the 2019 American Control Conference, Philadelphia, USA, pp. 4791 - 4796.

Xu Q, Li Y, Yu YH, Ding B, Jiang Z, Lin Z and Cazzolato B (2019) Experimental and numerical investigations of a two-body floating-point absorber wave energy converter in regular waves. Journal of Fluids and Structures : 102613. 